ми машинами кругового действия «Каскад» // Мелиорация. - 2019. - № 1(87). - С. 78-82.

3. Совершенствование технологии полива дождевальными машинами кругового действия «КАСКАД» / Д.А. Соловьев [и др.] // Научная жизнь. - 2019. - № 1.- С. 57-65.

4. Труфляк Е. В. Основные элементы системы точного земледелия. - Краснодар: КубГАУ, 2016. - 39 с.

Соловьев Дмитрий Александрович, $\partial-p$ mехн. наук, доцент, зав. кафедрой «Техносферная безопасность и транспортно-технологические машины», Саратовский государственный аграрный университет имени Н.И. Вавилова. Россия.
Журавлева Лариса Анатольевна, $\partial-p$ техн. наук, проф. кафедры «Техносферная безопасность и транспортно-технологические машины», Саратовский государственный аграрный университет имени Н.И. Вавилова. Россия.

410056, г. Саратов, ул. Советская, 60.

Тел.: (8452) 74-96-63.

Ключевъе слова: оросительный комплекс; дождевание; контроль и управление; цицфровые технологии; автоматизация; роботизация сельского хозяйства; ресурсосберегающие технологии.

\title{
ROBOTIC IRRIGATION COMPLEX “CASCADE”
}

Soloviev Dmitry Aleksandrovich, Doctor of Technical Sciences, Associate Professor, Head of the chair "Technosphere Safety and Transport-technological Machines", Saratov State Agrarian University named after N.I. Vavilov. Russia.

Zhuravleva Larisa Anatolyevna, Doctor of Technical Sciences, Associate Professor of the chair "Technosphere Safety and Transport-technological Machines", Saratov State Agrarian University named after N.I. Vavilov. Russia.

Keywords: irrigation complex; sprinkling; control and management; digital technologies; automation; robotization of agriculture; resource-saving technologies.

The article considers the possibility of improving irrigation technology by expanding the functions of the automation system, the introduction of digital technologies and intelligent-advising control systems. The developed control system installed on the irrigation complex "CASCADE" provides control of the irrigation complex and pumping station with the transmission of information to the operator panel, followed by processing and analysis of data. Made on the basis of a programmable logic controller allows you to ensure timely watering with the introduction of the necessary fertilizers and pesticides, control water consumption, fuel level in the generator set and other parameters. It is possible to configure SMS alerts on mobile phones of the staff. The implemented module of the intelligent-advising system allows changing the irrigation rate in accordance with the level of moisture reserves of the field areas in real time, based on the readings of sensors and weather stations, without human intervention

\section{АНАЛИЗ РЕЖИМОВ РАБОТЫ ПОТРЕБИТЕЛЕЙ СРЕДНЕГО И ВЫСОКОГО ДАВЛЕНИЯ ПРИ ОТКАЗАХ НА ГАЗОПРОВОДАХ}

\author{
СОЛОВЬЕВА Елена Борисовна, Национальный исследовательский Московский \\ государственный строительный университет
}

В работе рассмотрены методы проектирования кольцевого газопровода среднего и высокого давления с учетом требований надежности.

Введение. Главной задачей распределительной системы газоснабжения является ежечасовая подача газа потребителям. Кроме того, необходимо исключить возможность возникновения утечки газа из подземных газопроводов, которые могут при определенных условиях способствовать образованию газовоздушной смеси $[1,5]$. Системы газораспределния предназначены снабжать потребителей определенным количеством газа.

Основным критерием надежности систем газораспределения является вероятность их безопасной работы в течение заданного времени $P(t)$.

Отказ элементов систем газораспределения связан с возможностью возникновения значи- тельных трудностей для потребителей, особенно это сказывается при низких температурах наружного воздуха. При выходе из строя участка газопровода приходится отключать районные котельные, жилые дома, коммунальные предприятия, что приводит к ухудшению условий жизнедеятельности [4].

Поэтому при проектировании системы газораспределения необходимо предусмотреть варианты, которые бы снизили вероятность отключения потребителей от газопроводов [2, 3].

При проектировании систем газораспределения применяют следующие методы: переход от тупиковой к кольцевой сети; секционирование; 
дублирование; использование схем, исключающих отключение потребителей от систем газораспределения.

Методика исследований. Не все повреждения элементов систем газораспределения приводят к отключениям потребителей от газопровода. Большая часть повреждений на газопроводах ликвидируется без отключения потребителей. И только неисправности, связанные с немедленным выходом из строя элементов газораспределения, являются отказом.

Основными причинами, приводящими к отказам элементов систем газораспределения, являются механические повреждения; коррозионные неисправности; разрывы сварных швов.

Все эти неисправности, кроме механических повреждений, являются случайными и редкими событиями. Поэтому используются при анализе обеспеченности потребителей в аварийных режимах.

Рассмотрим вопрос о методах проектирования кольцевого газопровода среднего или высокого давления с точки зрения надежности работы. В качестве расчетной схемы примем кольцо, показанное на рис. 1 , которое имеет пять узловых нагрузок.

Результаты исследований. Диаметр кольца рассчитываем на $\frac{P_{\mathrm{H}}^{2}-P_{\mathrm{K}}^{2}}{=\text { const. }}$

Исходная формула ${ }^{l}$ для расчета гидравлических потерь:

$$
P_{\mathrm{H}}^{2}-P_{\mathrm{K}}^{2}=k \frac{1}{d^{5,25}} \cdot Q^{2}=a Q^{2},
$$

где $k$ - коэффициент, зависящий от свойств газа; $a$ - гидравлическое сопротивление газопровода.

Диаметр кольца $d_{0}$, длина каждого участка $l_{0}$. Так как размеры и геометрические формы

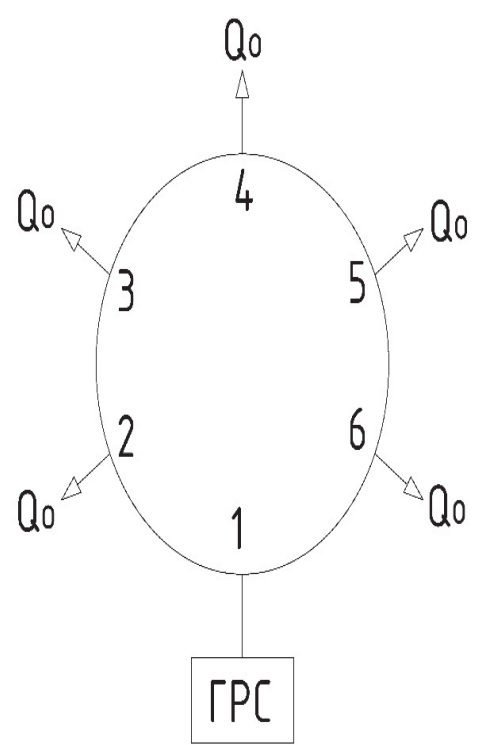

Рис. 1. Расчетная схема кольца высокого давления постоянного диаметра кольца, направление потоков и нагрузки симметричны, то гидравлическая увязка не требуется.

Потери давления в кольце при расчетной нагрузке:

$$
\begin{gathered}
P_{1}^{2}-P_{2}^{2}=a_{0}\left(0,5 Q_{0}\right)^{2} ; \\
P_{2}^{2}-P_{3}^{2}=a_{0}\left(1,5 Q_{0}\right)^{2} ; \\
P_{3}^{2}-P_{4}^{2}=a_{0}\left(2,5 Q_{0}\right)^{2} ; \\
P_{1}^{2}-P_{4}^{2}=P_{\mathrm{H}}^{2}-P_{\mathrm{k}}^{2}=8,75 a_{0} Q_{0}{ }^{2} \\
P_{1}^{2}-P_{4}^{2}=P_{\mathrm{H}}^{2}-P_{\mathrm{K}}^{2}=8,75 a_{0} Q_{0}{ }^{2} .
\end{gathered}
$$

Определим пропускную способность кольца при выключении наиболее невыгодного участка $1-2$.

Обозначим узловой расход при аварийном режиме в долях от $Q_{0}$.

$$
Q=x Q_{0} .
$$

Общая потеря давления:

$$
\begin{gathered}
P_{3}^{2}-P_{2}^{2}=a_{0}\left(x Q_{0}\right)^{2}=x^{2} a_{0} Q_{0}^{2} ; \\
P_{4}^{2}-P_{3}^{2}=a_{0}\left(2,0 x Q_{0}\right)^{2}=4 x^{2} a_{0} Q_{0}^{2} ; \\
P_{5}^{2}-P_{4}^{2}=a_{0}\left(3,0 x Q_{0}\right)^{2}=9 x^{2} a_{0} Q_{0}^{2} ; \\
P_{6}^{2}-P_{5}^{2}=a_{0}\left(4,0 x Q_{0}\right)^{2}=16 x^{2} a_{0} Q_{0}^{2} ; \\
P_{1}^{2}-P_{6}^{2}=a_{0}\left(5,0 x Q_{0}\right)^{2}=25 x^{2} a_{0} Q_{0}^{2} ; \\
P_{1}^{2}-P_{2}^{2}=\left(P_{\mathrm{H}}^{2}-P_{\mathrm{k}}^{2}\right)_{\text {обш }}=55 x^{2} a_{0} Q_{0}^{2} .
\end{gathered}
$$

Если начальное и конечное давление для расчетного и аварийного режимов одинаковое, тогда потребители при аварийном режиме работы сети смогут получить следующее количество газа:

$$
P_{\mathrm{r}}^{2}-P_{\mathrm{k}}^{2}=\left(P_{\mathrm{H}}^{2}-P_{\mathrm{k}}^{2}\right)_{\text {общ, }}
$$

или

$$
8,75 a_{0} Q_{0}^{2}=55 x^{2} a_{0} Q_{0}^{2} .
$$

Отсюда $x=\frac{8,75}{55}=0,39$, то есть составляет $39 \%$ расчетной величины.

Такую пропускную способность кольца при аварийном режиме работы следует признать недостаточной. Следовательно, кольцо высокого давления при принятых условиях расчета не обеспечивает надежность работы системы. Для обеспечения допустимого снижения пропускной способности кольца нужно иметь резерв давления.

Определим необходимый резерв давления для кольца среднего и высокого давления при условии обеспечения пропускной способности кольца при аварийном режиме, равной 0,8 расчетного значения. Необходимый резерв давления определяется условием 


$$
\frac{\left(P_{\mathrm{H}}^{2}-P_{\mathrm{K}}^{2}\right)_{\text {o6 }}}{\left(P_{\mathrm{H}}^{2}-P_{\mathrm{K}}^{2}\right)_{\mathrm{K}}}=\frac{55 \cdot 0,8^{2} a_{0} Q_{0}^{2}}{8,75 a_{0} Q_{0}^{2}},
$$

или

$$
\left(P_{\mathrm{H}}^{2}-P_{\mathrm{K}}^{2}\right)_{\text {общ }}=4,023\left(P_{\mathrm{H}}^{2}-P_{\mathrm{K}}^{2}\right) \text {. }
$$

Определим необходимый резерв давлений для кольца среднего давления:

$$
\begin{gathered}
P_{\mathrm{H}}=0,4 \mathrm{MПа;} P_{\mathrm{k}}=0,2 \text { Мпа; } \\
\left(P_{\mathrm{H}}^{2}-P_{\mathrm{K}}^{2}\right)_{\text {общ }}=0,4^{2}-0,2^{2}=0,12 \text { Мпа; } \\
\left(P_{\mathrm{H}}^{2}-P_{\mathrm{K}}^{2}\right)_{\mathrm{K}}=\frac{\left(P_{\mathrm{H}}^{2}-P_{\mathrm{K}}^{2}\right)_{\text {обш }}}{4,023}=\frac{0,12}{4,023}=0,029 .
\end{gathered}
$$

Величина расчетного перепада давления должна быть следующей:

$$
\begin{gathered}
P_{\mathrm{\kappa}}=\sqrt{0,4^{2}-0,029}=0,36 \mathrm{MПа;} \\
\Delta p_{\mathrm{p}}=0,4-0,36=0,04 \mathrm{M \Pi а}, \\
\text { или } \frac{0,04}{0,3} \cdot 100 \%=13,3 \%
\end{gathered}
$$

от избыточного давления в начале сети.

Принятый резерв давления позволяет увеличить перепад при аварийном режиме на $0,36-0,2=0,16$ Мпа.

Находим резерв давлений для кольца высокого давления:

$$
\begin{gathered}
P_{\mathrm{H}}=0,7 \mathrm{M \Pi а} ; P_{\mathrm{K}}=0,4 \mathrm{M} \Pi ; \\
\left(P_{\mathrm{H}}^{2}-P_{\mathrm{K}}^{2}\right)_{\text {общ }}=0,7^{2}-0,4^{2}=0,33 \mathrm{M} \text { Ма; } \\
\left(P_{\mathrm{H}}^{2}-P_{\mathrm{K}}^{2}\right)_{\mathrm{K}}=\frac{0,33}{4,023}=0,08 .
\end{gathered}
$$

Значение расчетного перепада давления:

$$
\begin{gathered}
P_{\mathrm{k}}=\sqrt{0,7^{2}-0,08}=0,64 \mathrm{MПа} . \\
\Delta p_{\mathrm{p}}=0,06 \mathrm{M \Pi а}, \frac{0,06}{0,6} \cdot 100 \%=10 \% .
\end{gathered}
$$

Рассмотрим работу кольца, имеющего те же геометрическую форму и размеры, те же нагрузки и расчетные перепады давления, но рассчитанного по $\frac{p_{R}^{2}-p_{R}^{2}}{l}=$ const. Длина участков одинакова, следовательно, для всех участков будет соблюдаться соотношение

$$
\begin{aligned}
& P_{1}^{2}-P_{2}^{2}=P_{2}^{2}-P_{3}^{2}=P_{3}^{2}-P_{4}^{2}= \\
& =P_{1}^{2}-P_{6}^{2}=P_{6}^{2}-P_{5}^{2}=P_{5}^{2}-P_{4}^{2} .
\end{aligned}
$$

Определим сопротивления участков:

$$
\begin{aligned}
& P_{3}^{2}-P_{4}^{2}=a_{3-4}\left(0,5 Q_{0}\right)^{2} ; \\
& P_{2}^{2}-P_{3}^{2}=a_{2-3}\left(1,5 Q_{0}\right)^{2} ;
\end{aligned}
$$

$$
P_{1}^{2}-P_{2}^{2}=a_{1-2}\left(2,5 Q_{0}\right)^{2} .
$$

Следовательно

$$
\begin{aligned}
& a_{1-2}\left(2,5 Q_{0}\right)^{2}=a_{2-3}\left(1,5 Q_{0}\right)^{2} ; \\
& a_{2-3}=\frac{6,25}{2,25} a_{1-2}=2,78 a_{1-2} ; \\
& a_{1-2}\left(2,5 Q_{0}\right)^{2}=a_{3-4}\left(0,5 Q_{0}\right)^{2} ; \\
& a_{3-4}=\frac{6,25}{0,25} a_{1-2}=25 a_{1-2} .
\end{aligned}
$$

Учитывая полученные соотношения сопротивлений участков, рассчитаем аварийный режим:

$$
\begin{gathered}
P_{2}^{2}-P_{1}^{2}=a_{1-2} ; \\
P_{3}^{2}-P_{2}^{2}=a_{2-3}\left(x Q_{0}\right)^{2}= \\
=x Q_{0}^{2} \cdot 2,78 a_{1-2}=2,78 a_{1-2} Q_{0}^{2} ; \\
P_{4}^{2}-P_{3}^{2}=a_{3-4}\left(2 x Q_{0}\right)^{2}= \\
=4 x Q_{0}^{2} \cdot 25 a_{1-2}=100 a_{1-2} Q_{0}^{2} ; \\
P_{5}^{2}-P_{4}^{2}=a_{4-5}\left(3 x Q_{0}\right)^{2}= \\
=9 x Q_{0}^{2} \cdot 25 a_{1-2}=225 a_{1-2} Q_{0}^{2} ; \\
P_{6}^{2}-P_{5}^{2}=a_{5-6}\left(4 x Q_{0}\right)^{2}= \\
=16 x Q_{0}^{2} \cdot 2,78 a_{1-2}=44,48 a_{1-2} Q_{0}^{2} ; \\
P_{1}^{2}-P_{6}^{2}=a_{1-6}\left(5 x Q_{0}\right)^{2}=25 x Q_{0}^{2}=25 x Q_{0}^{2} ; \\
P_{1}^{2}-P_{2}^{2}=\left(P_{\mathrm{r}}^{2}-P_{\mathrm{k}}^{2}\right)_{\text {обш }}=397,26 x^{2} a_{1-2} Q_{0}^{2} .
\end{gathered}
$$

Для сравнения различных вариантов надо сравнить расход металла при одинаковых диаметрах, выбранных по $\frac{p_{R}^{2}-p_{\mathrm{K}}^{2}}{l}=$ const. Сравнение надо сводить при одинаковой пропускной способности и равных суммарных перепадах давления.

Отсюда $\quad 55 x^{2} a_{0} Q_{0}^{2}=397,26 x^{2} a_{1-2} Q_{0}^{2}$;

$a_{1-2}=0,13 a_{0}$.

Определим диаметры:

$$
\begin{gathered}
a_{2-3}=2,78 a_{1-2} ; \\
a_{2-3}=2,78 \cdot 0,13=0,36 a_{0} ; \\
a_{3-4}=2,78 a_{1-2} ; \\
a_{3-4}=25 \cdot 0,13=3,25 a_{0} .
\end{gathered}
$$

Отсюда

$$
\begin{gathered}
d_{1-2}=\frac{l}{d_{1-2}^{5,25}} ; a_{0}=k \frac{l}{d^{5,25}} ; \\
\frac{d_{1-2}}{d_{0}}=\left(\frac{a_{0}}{a_{1-2}}\right)^{0,19} ; d_{1-2}=\frac{d_{0}}{0,13 a_{0}} ; \\
\frac{d_{2-8}}{d_{0}}=\left(\frac{a_{0}}{a_{2-8}}\right)^{0,19} ; d_{2-3}=1,21 d_{0} ;
\end{gathered}
$$




$$
\begin{aligned}
& \frac{d_{8-4}}{d_{0}}=\left(\frac{a_{0}}{a_{s-4}}\right)^{0,19} ; d_{3-4}=0,8 d_{0} ; \\
& \frac{d_{1-2}}{d_{0}}=\left(\frac{a_{0}}{a_{1-2}}\right)^{0,19} ; d_{1-2}=1,47 .
\end{aligned}
$$

Определим расход металла на кольцо. Расход металла на газопровод определим по уравнению

$$
M=\pi d_{i} \delta l \rho_{\mathrm{M}}=c l d,
$$

где $\delta$ - толщина стенки газопровода, она не зависима от диаметра; $\rho_{\text {м }}$ - плотность металла.

$$
c=\pi \delta \rho_{\mathrm{m}}=\text { const. }
$$

Так как все участки кольца имеют одинаковую длину, расход металла на участок определяется выражением

$$
M=c_{1} d_{0} .
$$

Расход металла на кольцо:

$$
M=c_{1} 2(1,21+0,8+1,47) d_{0}=3,48 c_{1} d_{0} .
$$

Нерасход металла по отношению к кольцу с постоянным диаметром составит

$$
\frac{3,48-6}{6}=\frac{2,52}{6}=0,42 \text {, или } 42 \% .
$$

Заключение. Таким образом, из сравнения рассмотренных вариантов можно сделать вывод, что при одинаковой гидравлической надежности кольцо с постоянным диаметром экономичности кольца, рассчитанного на постоянный период,
$\frac{p_{\mathrm{R}}^{2}-p_{\mathrm{K}}^{2}}{l}=$ const.

\section{СПИСОК ЛИТЕРАТУРЫ}

1. Национальный стандарт РФ ГОСТ Р 59 9612012 Системы газораспределительные. Сети газопотребления. Общие требования к эксплуатации // СПС «Гарант».

2. Приказ Ростехнадзора от 15.11.2013 № 542. «Об утверждении федеральных норм и правил в области промышленной безопасности» Правила безопасности сетей газораспределения и газопотребления. - Режим доступа: http://docs.cntd.ru/document/499061806.

3. СП 62.13330.2011 Газораспределительные системы. - Режим доступа: http://docs.cntd.ru/ document $/ 1200084535$.

4. Zhila V., Solovyeva E. The main directions of increasing the reliability of gas distribution systems in highrise districts // MATEC Web of Conferences, 2018, Vol. $170,03016$.

5. Solovyeva E.B. Evaluation of the safety of gas distribution systems, taking into account potential gas leakage from gas pipelines of high and medium pressure // International Journal of Mechanical Engineering \& Technology (IJMET), 2018, Vol. 9, Iss. 2, P. 761-764.

Соловьева Елена Борисовна, канд. техн. наук, доцент кафедры «Теплогазоснабжение и вентилящия», Национальный исследовательский Московский государственный строительный университет, Россия.

129337, г. Москва, Ярославское шоссе, 26.

Тел.: (495) 781-80-07.

Ключевые слова: высокое давление; среднее давление; газопровод; отказ.

\section{ANALYSIS OF THE OPERATING MODES OF MEDIUM AND HIGH PRESSURE CONSUMERS IN CASE OF GAS PIPELINE FAILURES}

Solovyova Elena Borisovna, Candidate of Technical Sciences, Associate Professor of the chair "Heat and Gas Supply and Ventilation", National Research Moscow State University of Civil Engineering. Russia.
Keywords: high pressure; average pressure; gas pipeline; failure.

This paper discusses design methods for a medium and high pressure ring gas pipeline taking into account reliability requirements. 